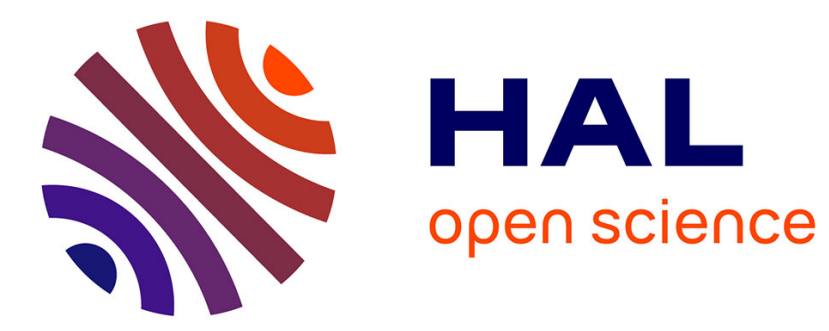

\title{
When Organometallic Chemistry and Metal Oxide Nanoparticles Meet Optimized Silicon-based Gas Sensor
}

N'Goran Pamela Yoboue, Philippe Menini, André Maisonnat, Myrtil L. Kahn, Katia Fajerwerg, Bruno Chaudret, Pierre Fau

\section{- To cite this version:}

N'Goran Pamela Yoboue, Philippe Menini, André Maisonnat, Myrtil L. Kahn, Katia Fajerwerg, et al.. When Organometallic Chemistry and Metal Oxide Nanoparticles Meet Optimized Silicon-based Gas Sensor. MRS Proceedings, 2011, 1253, 10.1557/PROC-1253-k05-18 . hal-02072230

\section{HAL Id: hal-02072230 \\ https://hal.laas.fr/hal-02072230}

Submitted on 19 Mar 2019

HAL is a multi-disciplinary open access archive for the deposit and dissemination of scientific research documents, whether they are published or not. The documents may come from teaching and research institutions in France or abroad, or from public or private research centers.
L'archive ouverte pluridisciplinaire HAL, est destinée au dépôt et à la diffusion de documents scientifiques de niveau recherche, publiés ou non, émanant des établissements d'enseignement et de recherche français ou étrangers, des laboratoires publics ou privés. 


\section{When organometallic chemistry and metal oxide nanoparticles meet optimized silicon based gas sensor}

Paméla Yoboue ${ }^{1}$, Philippe Ménini ${ }^{1,2}$, André Maisonnat ${ }^{3}$, Myrtil Kahn ${ }^{3}$, Katia Fajerwerg ${ }^{2,3}$, Bruno Chaudret $^{3}$, Pierre $\mathrm{Fau}^{2,3}$

${ }^{1}$ Laboratoire d'Analyse et d'Architecture des Systèmes, LAAS-CNRS, 7 avenue du colonel Roche, 31077 Toulouse, France

${ }^{2}$ University of Toulouse (Toulouse III), 118 route de Narbonne, 31062 Toulouse Cedex 02, France

${ }^{3}$ Laboratoire de Chimie de Coordination LCC-CNRS, 205 route de Narbonne, 31077 Toulouse Cedex 04, France

\section{ABSTRACT}

A robust silicon gas sensor chip (platinum heater, low stress membrane) has been designed and successfully operated with various metal oxide nanoparticles synthesized by organometallic route $\left(\mathrm{SnO}_{2}, \mathrm{ZnO}\right)$ deposited by a generic ink jet method. High quality and micron thick layers can be obtained and gas sensitivity (under $\mathrm{CO}$ ) is presented.

\section{INTRODUCTION}

Air quality control in confined places (automotive cabin, transportation and offices or working places) is getting more and more pregnant in the actual pollution levels due to new industrial environment. Despite the intense research work in the field of new silicon sensors and sensitive elements, the need for a high accuracy and low cost metal oxide gas sensors remains a challenge. Generally, the gas sensor substrate is based on commercial alumina support which comprises sensitive layer contact electrodes and an integrated platinum heater for heating purpose. These devices present high power consumption and are not adapted for devices miniaturization. Since few years, an alternative to alumina substrate is brought by microelectronics processes with the development of micromachined silicon devices together with polysilicon heaters. The generally admitted drawbacks of silicon chip gas sensors is due to resistance drift of the polysilicon heating element, harsh mechanical stress of the thermally insulating membrane and deactivation of the thin oxide sensitive layer. All the defects can lead to the progressive lost of sensor performance upon time. We have developed a new generation of metal oxide gas sensor, based on the combination of optimized micromachined silicon substrates and highly sensitive nanosized metal oxides derived from organometallic synthesis route [1, 2]. An integrated approach of nanoparticles combination with silicon based structures has been employed [3]. A platinum heater structure will insure very stable thermal performances of the device. This new sensor design has been built in accordance with the upstream constraints coming from the deposition method required by the liquid nature of the nano-sized sensitive material.

\section{EXPERIMENTAL}




\section{$\underline{\text { Silicon platform }}$}

Our SGS (semiconducting gas sensor) device is a global system that operates a high performance silicon micromachined substrate and a high sensitivity and stability semiconducting oxide. We present here a specific design which has been set up for the development of an optimized gas sensor adapted to sensitive layers prepared in a liquid form (colloidal solutions). The improvements of such silicon platform are (figure 1a):

- Small size: reduced sensitive layer area (200x200 microns).

- Interdigitated electrodes: compatible with high resistance sensitive layers (nano-sized oxides).

- Thermo-mechanical performance: low thermal conductivity and low stress $\mathrm{SiO}_{2}-\mathrm{SiN}_{\mathrm{x}}$ insulating membrane.

- Specific round shape insulating membrane: this is achieved by a deep reactive ion etching (DRIE) of the silicon backside. This shape allows a very low membrane bending upon heating.

- Reliability: robust miniaturized platinum heater meander, with distinct tracks for heater and measurement area. A micron thick silicon oxide layer in between the two platinum layers insures the electrical insulation.
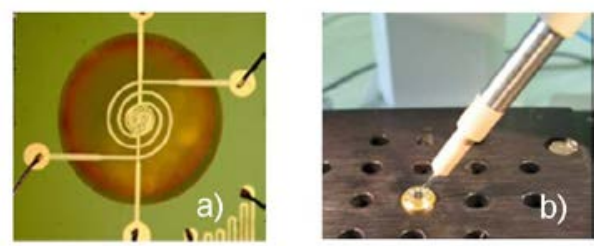

Figure 1: a) Silicon platform with integrated platinum heater and round shaped membrane for liquid implementation of the sensitive layer and b) drop deposition on by an ink-jet system.

\section{$\underline{\text { Sensitive layers synthesis }}$}

The sensitive metal oxide materials for sensing layers are prepared by organometallic routes according to previously described procedures [4]. This non aqueous chemistry allows preparing highly controlled nanoparticles (low size dispersion) in very clean conditions and halide free metal precursors. The thermolysis of tin amidure $\left[\mathrm{Sn}\left((\mathrm{NMe})_{2}\right)_{2}\right]_{2}$ precursor performed in presence of ppm controlled amounts of water in anisole solvent leads to the formation of a collection of partially oxidized tin nanoparticles. The stabilization of the material is achieved by the growth of a protective oxide shell around the metallic tin core. The mean size of these tin/tin oxide nanoparticles is close to $20 \mathrm{~nm}$, i.e. which allow a high sensitivity taking into account the accepted $3 \mathrm{~nm}$ depletion layer depth for tin oxide [5]. The full oxidation of tin/tin oxide composite into pure and well crystallized tin dioxide nanoparticles is directly achieved on the sensor surface through in-situ heating at $500^{\circ} \mathrm{C}$ by the platinum heater means.

Zinc oxide nanoparticles formation relies on a different synthesis procedure although based on similar organometallic chemistry principle [2]. A solution of the dicyclohexylzinc $\left(\mathrm{ZnCy}_{2}\right)$ in tetrahydrofurane (THF) is reacted with controlled amounts of water (2 molar equivalents) in presence of alkyamine organic ligand (octylamine, 1 molar equivalent) at room temperature. Dicyclohexylzinc is spontaneously transformed in the reaction mixture into zinc oxide nanoparticles with zincite structure, stabilized by long alkyl chain ligands. Mean particles size is $3 \mathrm{~nm}$ in diameter (figure $6 \mathrm{~b}$ ) and they display a round shaped form. After evaporation of the THF solvent, the particles are re-suspended in anisole solvent for liquid dispense purpose. 


\section{Sensitive layer deposition}

In both cases, tin and zinc oxides colloidal solutions are implemented as organic inks within an ink jet deposition system (Microdrop AG. MD series). This system is adapted to the deposition of controlled number of drops down to few tens of microns in diameter with ADK401 pipette (figure 1b). A sequential deposition process (solvent evaporation/dispense) allow the formation of the desired oxide layer just in the center of the contact electrode of the silicon device.

\section{RESULTS AND DISCUSSION}

\section{Silicon platform characterization}

Commercial integrated heaters on silicon platforms are generally built with n-doped (phosphorus) or p-doped (boron) polysilicon layers. The desired resistance level of the polysilicon layer is achieved by the high temperature diffusion $\left(1000^{\circ} \mathrm{C}\right)$ of the doping elements inside the silicon grains. One of the major drawbacks of such device is linked to the progressive drift of their electrical properties upon sensor operation temperature. At a constant temperature above $350^{\circ} \mathrm{C}$, the doping elements start to diffuse out from the polysilicon grains to the grain boundary where they become useless. The net result at constant voltage rate is a decrease of the heating power of the device upon time giving rise to a decrease of target gas sensitivity. Platinum based heater structures offer a much better thermo-electrical behavior since their operational temperature can be adjusted up to $650^{\circ} \mathrm{C}$ [3] without noticeable change in heater resistance value with time. The thermal response of the heater is presented in figure $2 \mathrm{a}$ and $2 \mathrm{~b}$.
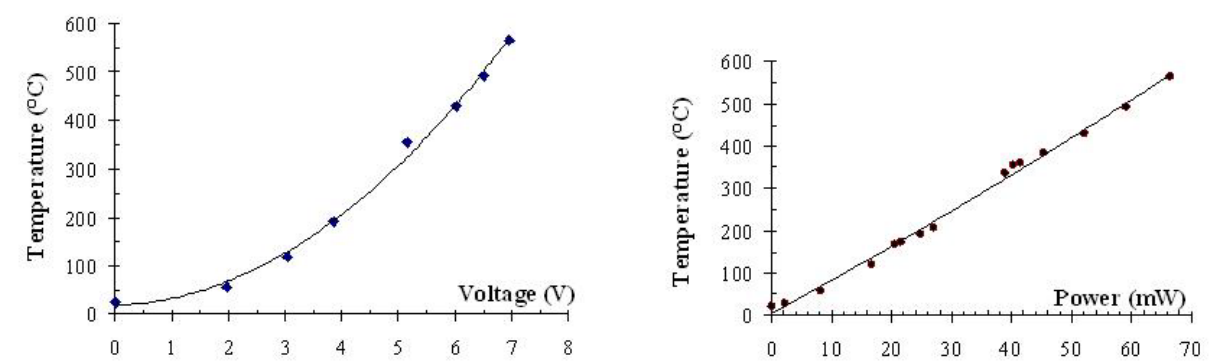

Figure 2: Temperature of a silicon device versus a) voltage and b) power (heater resistance 440 Ohms).

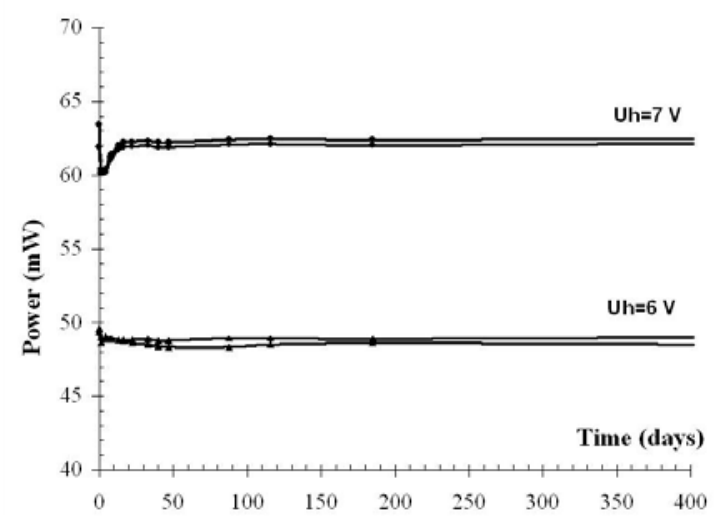

Figure 3: Power consumption stability of sensors operating at constant voltage 
The operational temperature is measured in the center of the sensitive layer area by a calibrated infra-red camera. A mean temperature of $500^{\circ} \mathrm{C}$ is obtained with a $6.5 \mathrm{~V}$ voltage at a power consumption of only $60 \mathrm{~mW}$. Constant temperature drift measurements of this device have been evaluated over a period of more than 400 days (figure 3). It appears that this heater element is very stable over such a period and even at very high temperature close to $600^{\circ} \mathrm{C}$.

The thermally insulating membrane with a rounded shape brings a better mechanical behavior to the sensor (figure 4).

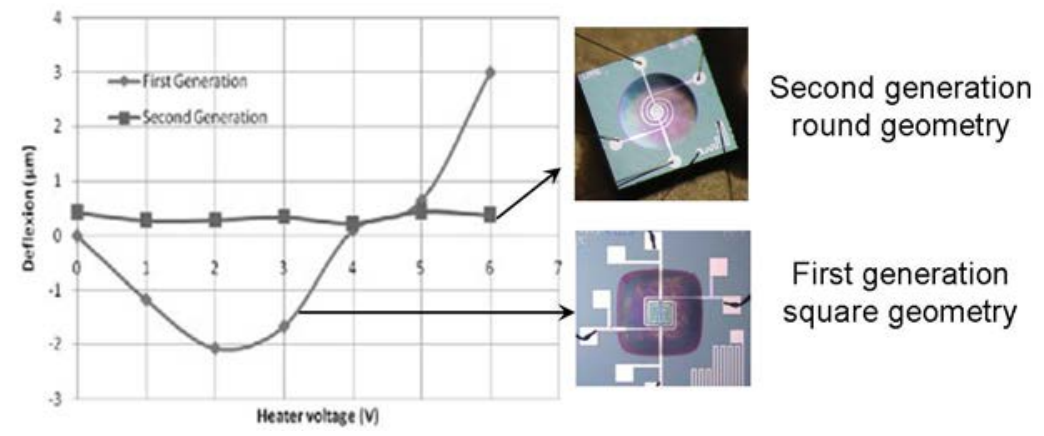

Figure 4: Membrane deformation with heater voltage in the case of first generation sensor with a rectangular shape and second generation with rounded geometry.

Previous geometries based on square or rectangular membrane generates a high level of stress resulting in large deformations of the structure upon temperature operation (from -2 to +3 micron height). Membrane deformation of round membranes remains limited to less than 1 micron height at a temperature of $400^{\circ} \mathrm{C}$ (voltage $6 \mathrm{~V}$ ). This will give rise to low induced stress in the deposited sensitive layers and therefore participates to the reliability of the device.

\section{Drop deposition of sensitive layers}

Metal oxide nanoparticles are obtained in organic solvent medium and therefore used as organic inks by a classical ink jet deposition system. The tip diameter and piezo actuated device allow the precise control of very small droplets containing metal oxide nanoparticles. The deposition process is based on a repetitive sequence of drop deposition, solvent evaporation and further drop deposition. The final sensitive layer thickness is micron large (figure $5 \mathrm{a}$ and $\mathrm{b}$ ) and a controlled thermal treatment up to $500^{\circ} \mathrm{C}$ of the sensitive layer is operated through the integrated heater. This final sensor preparation allows cleaning the sensitive layer by removing all traces of residual organics in the layer (solvent, and by-products from the nanoparticles synthesis).
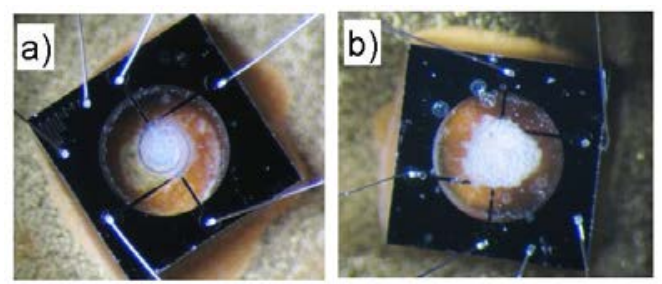

Figure 5: a) Optical images of a) tin dioxide b) zinc oxide layers on a silicon platform 
Figure 6a is a scanning electron microscopy (SEM) image of the final thick tin dioxide sensitive layers. It reveals the highly porous nature of the layer which is a consequence of the used deposition technique. Indeed, liquid deposition process induces large amounts of solvents as a carrier medium with the nanoparticles, leading to an important release of porosity in the deposited layers due to solvent evaporation. Final foam-like structure with high degree of open porosity is obtained and will give rise to large gases diffusion pathways inside the sensing layer. Figure 6b is a SEM image of the drop deposition of a very thin layer of colloidal zinc oxide on silicon substrates and shows up the regular pavement of the surface by nano $\mathrm{ZnO}$ particles.
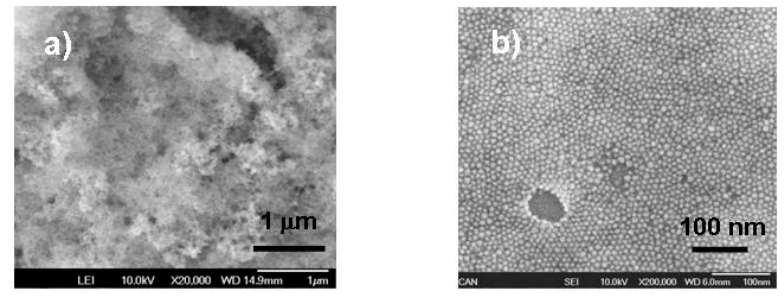

Figure 6: SEM images of a) highly porous 1 micron thick tin dioxide layer made of $20 \mathrm{~nm}$ large nanoparticles ( $\mathrm{G}=\mathrm{x} 20$ ’000), and b) zinc oxide nanoparticles thin layer deposited on silicon substrate (G=x200’000).

\section{$\underline{\mathrm{CO}}$ sensitivity of $\mathrm{SnO}_{2}$ and $\mathrm{ZnO}$ nanostructured layers}

Tests under gases have been performed in an automated test bench with nine available position tests in parallel. For this first attempt, standard test conditions have been set up and no determination of optimized parameters were conducted. The temperature of the sensitive layers (tin dioxide and zinc oxide) is maintained at $500^{\circ} \mathrm{C}$ (i.e. $6.5 \mathrm{~V}$ operating voltage), relative humidity is hold at a constant level of $50 \%$ and CO contents of 50, 200 and 500 ppm in air atmosphere are tested.
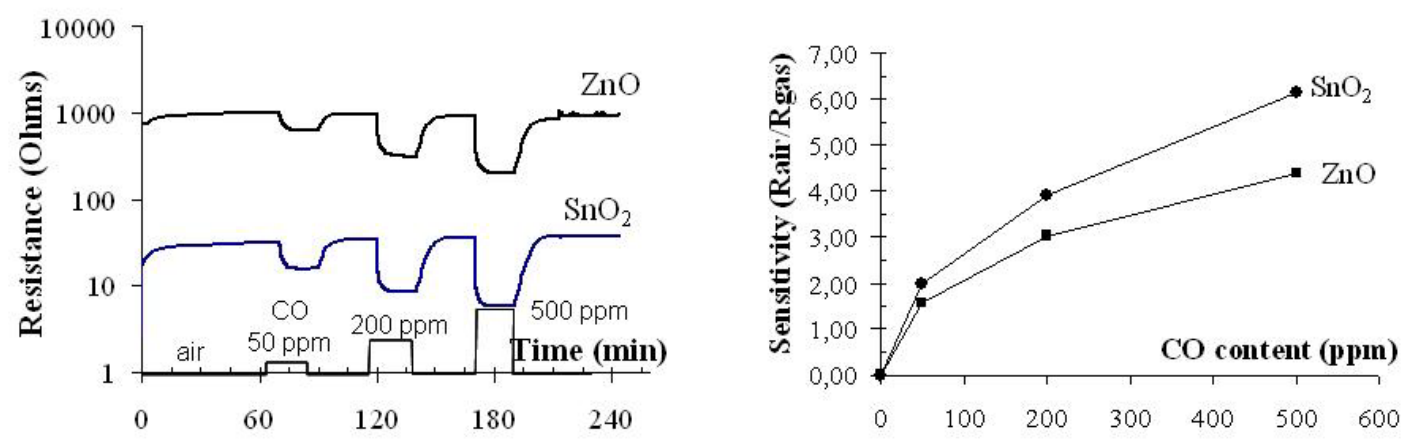

Figure 7: a) CO response of nano tin and zinc oxides and b) Sensitivity values for increasing CO exposure

An initial stabilization period of 8 hours under operating conditions (air, 50\% HR) is necessary before the sensor signal reaches a stable baseline. Once achieved, the sensors stabilization time are much shorter (less than an hour of air stabilization). Figure 7a presents the response of both zinc and tin dioxide gas sensors upon increasing CO levels. The first observation is the large difference in sensitive layer resistances under air. Zinc oxide sensor resistance is close to 1 Mohms whereas tin dioxide is only 30KOhms. These two materials have a similar band gap 
width $\left(3,37 \mathrm{eV}\right.$ for $\mathrm{ZnO}$ versus 3,5eV for $\left.\mathrm{SnO}_{2}\right)$, and are contacted by the same interdigitated platinum contact geometry. The smaller size of zinc oxide grains ( $3 \mathrm{~nm}$ ) compare to tin oxide (20 $\mathrm{nm})$ can be a part of the explanation for the very high resistance level observed for zinc oxide. In fact, depletion layers width are supposed to extend up to few nanometers (1-3 nm for zinc oxide according to Takahashi [6]). Therefore, this nano-sized zinc oxide layer presents the case of an almost fully electron-depleted oxide grains. A key parameter for an improved response of the sensor is the highly porous nature of the sensitive layer and the number of electrical contact between oxide grains. The sensitivity level is measured as the ratio of air resistance on gas resistance ( $\mathrm{R}$ air/ $\mathrm{R}$ gas). Despite a much lower grain size, zinc oxide presents a lower sensitivity to $\mathrm{CO}$ gas exposure than tin dioxide (figure $7 \mathrm{~b}$ ). Sensitivity is a very complex parameter and cannot be simply rely on physical parameters like the size of grains and width of depletion layer. Gas sensors are governed by complex catalytic reactions and the chemical nature of the grain surface, crystallographic orientation, grain boundaries or intra-grains neck formation, metal electrode/oxide grains junctions, are as well key parameters for gas reactions with oxide surfaces. This catalytic point of view of SGS devices is a part of our actual research areas on new gas sensors. Finally, undoped nanosized tin oxide sensors present a very high sensitivity (R air / $\mathrm{R} \mathrm{CO}=6)$ to $500 \mathrm{ppm} \mathrm{CO}$. This is a promising result in view of a future improvement of the sensitive layers through doping by metal catalysts or mixing with other nanosized metal oxides. New tests on nano $\mathrm{ZnO}$ and $\mathrm{SnO}_{2}$ are currently under investigation.

\section{CON CLUSION}

We have presented the development of an optimized silicon based SGS gas sensor working with micron thick layers of tin and zinc oxides nanoparticles. Drop deposition technique is a powerful way in order to prepare sensitive layers on narrow contact electrodes on silicon surfaces. All our future developments will be done in combination with the reliable, electrically stable and low power consumption silicon device described in this paper.

\section{ACKNOWLEDGMENTS}

The authors thank CNRS and University Paul Sabatier for its support. We also gratefully thank the TEMSCAN service and especially M. Vincent Collière for SEM images.

\section{REFERENCES}

[1] C. Nayral et al., App. Surf. Sci., 2000, 164, 219 - 226.

[2] M. Monge, M. L. Kahn, A. Maisonnat, B. Chaudret, Angew. Chem. Int. Ed., 2003, 42, 53215324.

[3] Ph. Ménini et al., Eurosensors XXII proceedings, 2008, 342.

[4] C. Nayral et al. Adv. Mater. 1999, 11, No. 1, 61-63

[5] N. Yamazoe, Sens. and act., 2005, B 108, 2-14.

[6] Y. Takahashi et al. Jpn. J. App. Phys., 1994, vol. 33, 6611-6615 